\title{
FÓRUM
}

Submetido 18.06.2020. Aprovado 18.11.2020

Avaliado pelo sistema double blind review. Editores convidados: Alessandra de Sá Mello da Costa, Ely Laureano Paiva, Marcus Vinícius Peinado Gomes e Vinicius Brei

Versão original

DOI: http://dx.doi.org/10.1590/So034-759020200604

\section{EFEITOS DA INFORMAÇÃO NA INSEGURANÇA E ENGAJAMENTO NO TRABALHO EM TEMPOS DE PANDEMIA}

\author{
Effects of information on job insecurity and work engagement in times of pandemic \\ Efectos de la información sobre la inseguridad y compromiso laboral en tiempos \\ de pandemia
}

\begin{abstract}
RESUMO
Pandemias suscitam cenários de incerteza que afligem até mesmo pessoas acostumadas a promover congruência de informações, como em empresas fintechs. Assim, objetiva-se investigar os efeitos do compartilhamento vertical da informação na mitigação da insegurança no trabalho e no engajamento no trabalho, moderada pela credibilidade da informação de mídias sociais sobre pandemia. Uma survey foi realizada com funcionários da maior startup financeira brasileira e, na análise dos dados, aplicou-se modelagem de equações estruturais e fuzzy-set QCA. Os resultados apontam que compartilhamento vertical da informação mitiga insegurança no trabalho e exerce efeitos no engajamento no trabalho. Compartilhamento vertical da informação e ausência da insegurança são, respectivamente, quase sempre e sempre necessárias, e suficientes para alto engajamento no trabalho. Ademais, a credibilidade da informação modera a relação entre insegurança e engajamento no trabalho. Esses resultados proporcionam contribuições ao revelar efeitos de antecedentes da insegurança e do engajamento no trabalho num período singular de pandemia.
\end{abstract}

PALAVRAS-CHAVE | Insegurança no trabalho, engajamento no trabalho, compartilhamento vertical da informação, credibilidade da informação, tempos de pandemia.

\section{ABSTRACT}

Pandemics raise scenarios of uncertainty that afflict even people who are used to promoting information congruence, as in fintechs. Thus, the purpose is to investigate the effects of vertical information sharing on mitigating job insecurity and on work engagement, moderated by the credibility of the social media information about the pandemic. A survey was carried out with employees of the largest Brazilian financial startup and structural equation modeling and fuzzy-set QCA were applied to the data analysis. The results show that vertical information sharing mitigates job insecurity and exerts effects on work engagement. Vertical information sharing and absence of job insecurity are, respectively, almost always and always necessary, and sufficient for high work engagement. Furthermore, information credibility moderates the relationship between job insecurity and work engagement. These results provide contributions by revealing effects that are antecedents of job insecurity and work engagement in a unique period of pandemic.

KEYWORDS / Job insecurity, Work engagement, Vertical information sharing, Information credibility, times of pandemic.

\section{RESUMEN}

Las pandemias plantean escenarios de incertidumbre que afectan incluso a las personas acostumbradas a promover la congruencia de la información, como en las empresas fintech. Así, el objetivo es investigar los efectos de la compartición vertical de información para mitigar la inseguridad laboral y garantizar el compromiso en el trabajo, moderada por la credibilidad de la información de las redes sociales sobre la pandemia. Se realizó una encuesta con empleados de la mayor startup financiera brasileña y en el análisis de datos, se aplicó el modelado de ecuaciones estructurales y fuzzy-set QCA. Los resultados muestran que la compartición vertical de información mitiga la inseguridad laboral y ejerce efectos sobre el compromiso laboral. La compartición vertical de información y la ausencia de inseguridad son, respectivamente, casi siempre y siempre necesarios, y suficientes para una alta participación en el trabajo. Además, la credibilidad de la información modera la relación entre inseguridad y compromiso en el trabajo. Estos resultados proporcionan contribuciones al revelar los efectos de fondo de la inseguridad y el compromiso laboral en un período único de pandemia.

PALABRAS CLAVE I Inseguridad laboral, Compromiso laboral, Compartición vertical de información, Credibilidad de la información,tiempos de pandemia. 


\section{INTRODUÇÃO}

As turbulências econômicas, sociais e psicológicas decorrentes da pandemia de Covid-19 (Goodell, 2020; Kuckertz et al., 2020) levam os indivíduos a enfrentar níveis mais agudos de insegurança no trabalho (Hamouche, 2020), o que pode resultar em menor engajamento (Saks, 2006). Nesse cenário, o compartilhamento vertical da informação pode proporcionar maior interação entre gestores e funcionários (Parker \& Kyj, 2006), seja de informações financeiras ou não financeiras (Macinati, Bozzi, \& Rizzo, 2016), e contribuir para minimizar a insegurança e promover o engajamento no trabalho (Baker, 2020; Bao, Sun, Meng, Shi, \& Lu, 2020; Blake, Blendon, \& Viswanath, 2010; Hamouche, 2020; Huang, Niu, Lee, \& Ashford, 2012; Men \& Yue, 2019; Mishra, Boynton, \& Mishra, 2014; Vander Elst, Baillien, Cuyper, \& Witte, 2010; Verčič, Verčič, \& Sriramesh, 2012; Welch, 2012).

Especula-se que a (não) credibilidade da informação sobre a pandemia de Covid-19, pautada principalmente nas mídias sociais, pode atenuar os efeitos do compartilhamento vertical da informação e da insegurança no trabalho no engajamento no trabalho. Tal presunção decorre de as mídias sociais representarem um dos principais e mais atualizados meios de comunicação sobre a pandemia de Covid-19 (Bao et al., 2020). Destacam-se, no entanto, pela divulgação de informações enganosas (Gao et al., 2020), o que interfere na comunicação e compartilhamento das informações nas organizações (Hamouche, 2020; Karasek, 1979; Welch, 2012), e pode agravar a insegurança no trabalho (Blake et al., 2010; Hamouche, 2020), fatores esses que refletem no engajamento (Saks, 2006).

Diferentemente de estudos pregressos que analisaram antecedentes da insegurança e engajamento no trabalho, nesta pesquisa do tipo single entity, analisa-se um mecanismo gerencial que pode subsidiar a gestão (compartilhamento vertical da informação), além de considerar reflexos da credibilidade da informação das mídias sociais no emergente contexto da pandemia em uma startup. Em geral, startups possuem dificuldades de gestão (Carraro, Meneses, \& Brito, 2019), as quais se agravaram com os efeitos da pandemia de Covid-19 (Goodell, 2020; Kuckertz et al., 2020). Nesse sentido, objetiva-se investigar os efeitos do compartilhamento vertical da informação na mitigação da insegurança e no engajamento no trabalho, moderada pela credibilidade da informação (mídias sociais). As análises são desenvolvidas por meio das técnicas de modelagem de equações estruturais com estimação por mínimos quadrados parciais (SEM-PLS) e análise qualitativa comparativa fuzzy-set (fsQCA), visto que podem apresentar resultados complementares
(Crespo, Rodrigues, Samagaio, \& Silva, 2019; Kaya, Abubakar, Behravesh, Yildiz, \& Mert, 2020).

A relevância do estudo está no seu alinhamento com a lacuna apontada em pesquisas anteriores quanto à limitação de evidências acerca de mecanismos que atuem na mitigação da insegurança no trabalho (Darvishmotevali \& Ali, 2020; Karatepe, Rezapouraghdam, \& Hassannia, 2020; Richter \& Näswall, 2019). Com a inclusão do compartilhamento vertical da informação, pretende-se compreender como esse mecanismo gerencial atua em detrimento da insegurança e em prol do engajamento no trabalho, neste singular período da pandemia de Covid19 (Hamouche, 2020) e com disseminação de informações enganosas nas mídias sociais, que podem ser prejudiciais à saúde mental dos funcionários (Bao et al., 2020; Gao et al., 2020). O campo de investigação justifica-se pela capacidade de as startups inovarem e moldarem o futuro econômico (Walsh \& Cunningham, 2016), além de a pandemia de Covid19 ser uma ameaça à continuidade no mercado e ao potencial inovador (Kuckertz et al., 2020). Investigam-se os funcionários da maior startup brasileira de serviços financeiros, visto que estão acostumados ao desafio de promover a congruência de informações.

0 estudo contribui pelo fato de investigar o compartilhamento vertical da informação como possível antecedente da mitigação da insegurança e promotor do engajamento no trabalho, e também por verificar se a credibilidade da informação (mídias sociais) sobre a pandemia de Covid-19 auxilia o impacto do compartilhamento vertical da informação e insegurança em relação ao engajamento no trabalho. Contribuições gerenciais decorrem do estudo ao fornecer subsídios para que os gestores de startups, como no caso da fintech investigada, possam assegurar a continuidade do negócio, por meio de mecanismos que consigam manter os funcionários engajados no trabalho e sem decréscimo na produtividade.

\section{FRAMEWORK TEÓRICO}

\section{Insegurança e engajamento no trabalho}

Na literatura sobre insegurança no trabalho, as discussões são enraizadas no sentimento de impotência dos funcionários para garantir a continuidade almejada no seu trabalho, em decorrência de uma situação ameaçadora (Greenhalgh \& Rosenblatt, 1984). Devido à pandemia de Covid-19, diversas empresas, especialmente as inovadoras, como no caso de startups, têm sua sobrevivência ameaçada (Kuckertz et al., 2020), o que reflete no agravamento 
da saúde mental dos funcionários, de maneira a aumentar a insegurança e diminuir o engajamento no trabalho (Baker, 2020; Blake et al., 2010; Hamouche, 2020). Para Saks (2006), essa insegurança possui potencial de diminuir vigor, dedicação e estado de espírito positivo, ou seja, o engajamento no trabalho.

A literatura prévia sobre insegurança no trabalho aponta que esta tende a diminuir o engajamento no trabalho, fenômeno investigado em diferentes contextos, como o de trabalhadores italianos (Guarnaccia, Scrima, Civilleri, \& Salerno, 2018), funcionários expatriados de diferentes empresas da Arábia Saudita (Ali, Ali, Albort-Morant, \& Leal-Rodríguez, 2020; Ali, Ali, Grigore, Molesworth, \& Jin, 2020) e funcionários de hotéis internacionais da China (Karatepe et al., 2020). Foi observado, ainda, que funcionários os quais percebem altos níveis de insegurança no trabalho refletem em menor nível de engajamento (Stander \& Rothmann, 2010). Com base no exposto, presume-se que:

H1: Existe efeito negativo direto da insegurança no trabaIho no engajamento no trabalho.

Embora exista ampla sustentação na literatura para a associação negativa proposta entre insegurança e engajamento no trabalho, particularmente no campo de startups, e principalmente no contexto da pandemia de Covid-19, os achados ainda são limitados.

\section{Compartilhamento vertical da informação, insegurança e engajamento no trabalho}

A comunicação interna da organização configura-se como uma função gerencial que engloba o processo da comunicação, de modo a abranger elementos que resultem no engajamento dos funcionários (Mishra et al., 2014; Verčič et al., 2012; Welch, 2012). Nessa perspectiva, a prática gerencial do compartilhamento vertical da informação pode desempenhar um papel fundamental nas organizações, particularmente no contexto singular da pandemia de Covid-19 (Hamouche, 2020).

O compartilhamento vertical da informação permite que gestores e funcionários promovam maior interação (Parker \& Kyj, 2006), tanto de informações financeiras quanto de informações não financeiras (Lau \& Roopnarain, 2014; Macinati et al., 2016), o que pode reduzir a assimetria informacional e a insegurança no trabalho (Darvishmotevali \& Ali, 2020; Lau \& Roopnarain, 2014; Nouri \& Parker, 1998; Penno, 1984), além de manter o funcionário imerso na organização e promover engajamento no trabalho (Baiman \& Evans, 1983; Karasek, 1979; Locke, Schweiger, \& Latham, 1986).
Estudos prévios apresentam evidências de que o compartilhamento vertical da informação se faz importante para o comprometimento organizacional (Parker \& Kyj, 2006). A comunicação interna entre gestores e funcionários pode envolver estes na organização, aumentando o seu engajamento no trabalho (Mishra et al., 2014). Assim, assume-se que:

$\mathrm{H}_{2}$ : Existe efeito positivo direto do compartilhamento vertical da informação no engajamento no trabalho.

Por outro lado, a comunicação interna e a participação organizacional dos funcionários podem mitigar a insegurança no trabalho, conforme evidências da pesquisa com funcionários de organizações belgas (Vander Elst et al., 2010). Semelhantemente, foi constatado que o compartilhamento de informações e dos processos da tomada de decisão entre gestores e funcionários pode minimizar a insegurança no trabalho, em uma amostra de funcionários de empresas da China (Huang et al., 2012).

Dessa forma, presume-se que essa insegurança na continuidade do trabalho, agravada por uma situação ímpar (Greenhalgh \& Rosenblatt, 1984), possa ser minimizada pela comunicação interna (Verčič et al., 2012), especificamente o compartilhamento vertical de informações (Parker \& Kyj, 2006), financeiras e não financeiras (Lau \& Roopnarain, 2014; Macinati et al., 2016), que abarquem o contexto da pandemia de Covid19 (Hamouche, 2020). Assim, espera-se que:

$\mathrm{H}_{2}$ : Existe efeito negativo direto do compartilhamento vertical da informação na insegurança no trabalho.

O compartilhamento vertical da informação (Parker \& Kyj, 2006), financeira e não financeira (Lau \& Roopnarain, 2014; Macinati et al., 2016), pode reduzir a assimetria informacional e a insegurança no trabalho (Lau \& Roopnarain, 2014; Nouri \& Parker, 1998; Penno, 1984), e, mediante a redução da insegurança, exercer efeito indireto no engajamento no trabalho (Parker \& Kyj, 2006). Pela comunicação (Mishra et al., 2014) e compartilhamento vertical da informação (Parker \& Kyj, 2006), envolvem-se os funcionários na organização, o que constitui uma função gerencial pertinente para promover o engajamento no trabalho (Verčič et al., 2012; Welch, 2012) na pandemia de Covid-19 (Hamouche, 2020).

Assim como foi proposto o efeito positivo do compartilhamento vertical da informação no engajamento no trabalho $\left(\mathrm{H}_{2}\right)$ e negativo na insegurança no trabalho $\left(\mathrm{H}_{2}\right)$, além desta de maneira negativa no engajamento no trabalho $\left(\mathrm{H}_{1}\right)$, especula-se que o compartilhamento vertical da informação exerça efeito indireto positivo no engajamento no trabalho, mesmo por meio da insegurança no trabalho. Prevê-se mediação da insegurança no trabalho entre o compartilhamento vertical 
da informação e o engajamento no trabalho. Presume-se que o compartilhamento vertical da informação (Parker \& Kyj, 2006) promova efeitos indiretos no engajamento no trabalho (Saks, 2006), mesmo diante da insegurança no trabalho (Greenhalgh \& Rosenblatt, 1984) revelada no período de pandemia de Covid19 (Kuckertz et al., 2020). Assim, propõe-se que:

$\mathrm{H}_{\mathrm{c}}$ : Existe efeito indireto positivo do compartilhamento vertical da informação no engajamento no trabalho por meio da insegurança no trabalho.

\section{O papel moderador da credibilidade da informação nas mídias sociais}

As mídias sociais promovem a interação entre as pessoas, tendo seu uso impulsionado em tempos de distanciamento social, como no caso da pandemia de Covid-19 (Wiederhold, 2020). No entanto, teme-se por informações enganosas (fake news) constantemente disseminadas entre os seus usuários (Yanagi, Tahara, Orihara, Sei, \& Ohsuga, 2020). Para as fake news sobre a pandemia de Covid19, foi criado um termo próprio, a infodemic (Zarocostas, 2020), e elas podem promover o crescimento do surto e o processo epidêmico (Kim, Fast, \& Markuzon, 2019).

As mídias sociais representam uma das principais fontes de notícias atualizadas sobre a pandemia de Covid-19 (Bao et al., 2020), porém diversas informações compartilhadas podem ser enganosas ou equivocadas (Gao et al., 2020). A sobrecarga de informações nas mídias sociais, sendo várias delas infundadas e incrediveis (Bontcheva, Gorrell, \& Wessels, 2013), pode intensificar o medo (Hamouche, 2020), o estresse (Brooks, Dunn, Amlôt, Rubin, \& Greenberg, 2018), a ansiedade (Shigemura, Ursano, Morganstein, Kurosawa, \& Benedek, 2018), problemas na saúde mental (Gao et al., 2020), a insegurança no trabalho (Blake et al., 2010; Hamouche, 2020), além de interferir na comunicação e compartilhamento das informações nas organizações (Welch et al., 2012; Hamouche et al., 2020), fatores esses que refletem no engajamento no trabalho (Saks, 2006).

Em cenários com altas ou baixas percepções de credibilidade da informação sobre a pandemia de Covid-19, os efeitos do compartilhamento vertical da informação e da insegurança no trabalho no engajamento no trabalho podem repercutir de maneiras diferentes. Assume-se que exista moderação positiva nas relações supracitadas, já que quanto maior for a percepção da credibilidade da informação, maior será o engajamento no trabalho; por outro lado, quanto menor for essa, maiores serão os problemas psicológicos dos indivíduos (Blake et al., 2010; Brooks et al., 2018; Gao et al., 2020; Hamouche, 2020; Shigemura, Ursano,

Morganstein, Kurosawa, \& Benedek, 2020), logo será menor o engajamento no trabalho. Assim, assume-se que:

$\mathrm{H}_{3}$ : Existe efeito moderador positivo da credibilidade da informação (mídias sociais) na relação da insegurança no trabalho com o engajamento no trabalho.

$\mathrm{H}_{3}$ : Existe efeito moderador positivo da credibilidade da informação (mídias sociais) na relação do compartilhamento vertical da informação com o engajamento no trabalho.

Na Figura 1, apresenta-se o modelo teórico da pesquisa.

Figura 1. Modelo teórico

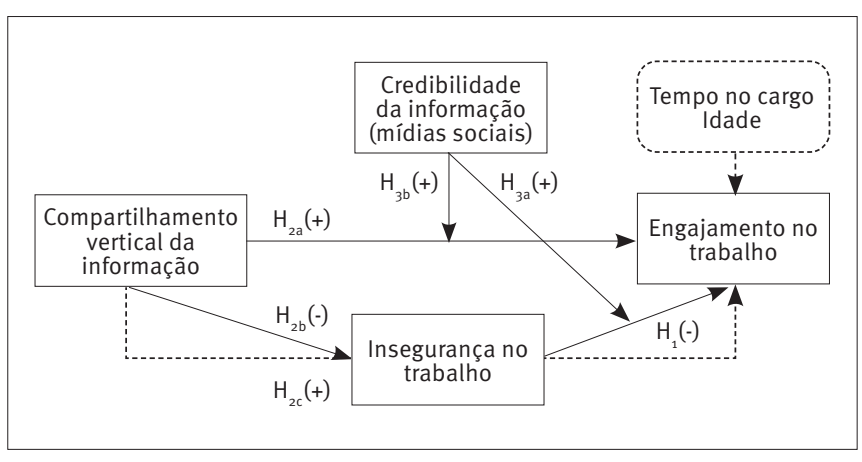

\section{METODOLOGIA}

\section{População e amostra}

Uma survey do tipo single entity foi realizada com funcionários da maior startup brasileira do segmento de serviços financeiros, considerando uma purposive non-probability sample. Uma purposive sample, também conhecida por judgmental ou expert sampling, figura como uma amostra não probabilística, que possui como objetivo central contemplar uma amostra que possa ser representativa da população, sendo adequada para uma definição populacional restrita e/ou determinada delimitação (Battaglia, 2008), no caso, uma single entity (fintech).

A escolha de uma fintech para o estudo de caso justifica-se pela escassez e consequente chamada para pesquisas com esse ramo de startups (Muthukannan, Tan, Gozman, \& Johnson, 2020), além de pela importância destas para a economia local e global (Haddad \& Hornuf, 2019). O caso investigado aparece no top 1 de startups brasileiras em diversos rankings, contudo mais informações não são fornecidas devido a termos éticos e anonimato.

Para contatar o público-alvo, fez-se uso da rede social Linkedin, que listava 2.648 funcionários dessa startup. Um 
convite foi enviado para todos e, aos 728 que aceitaram participar da pesquisa, na segunda mensagem foi informado o link do instrumento pela plataforma QuestionPro. Os contatos foram iniciados em 29 de abril de 2020 e os dados, coletados até 28 de maio do mesmo ano, totalizando 181 respostas completas, livres de possíveis outliers ou dados faltantes.

\section{Mensuração dos construtos}

Os construtos foram mensurados com escala tipo Likert de sete pontos ( $1=$ discordo totalmente e $7=$ concordo totalmente). 0 compartilhamento vertical da informação contém quatro indicadores, adaptados de Parker e Kyj (2006), relevando-se o contexto de informações financeiras e não financeiras. 0 construto da insegurança no trabalho compõe-se de quatro itens, adaptados de Witte (2000) e Darvishmotevali e Ali (2020). $O$ engajamento no trabalho compreende cinco assertivas, adaptadas de Saks (2006) e Ali, Ali, Albort-Morant e LealRodríguez (2020). A credibilidade da informação sobre a pandemia de Covid-19 relativa à comunicação externa (mídias sociais) compõe-se de três itens, adaptados de Thompson, Wang e Daya (2019).

Foram consideradas duas variáveis de controle relativas aos funcionários, transformadas em subgrupos para sua operacionalização: tempo no cargo atual («1ano e $\geq 1$ ano) e idade ( 30 anos e $\geq 30$ anos). Pesquisas anteriores nesse contexto controlaram pela variável idade do indivíduo (Lübke, 2019). Para a divisão dos grupos, foi tomada como parâmetro a pesquisa de Rai, Ghosh, Chauhan e Mehta (2017), na qual as duas faixas etárias ( 130 anos ou $\geq 30$ anos) demonstraram-se pertinentes. Quanto ao tempo no cargo atual, devido à startup em questão estar no mercado há menos de oito anos e ter ampliado o seu quadro funcional no último ano, essa variável foi considerada observando-se esse ponto de corte.

Devido ao fato de todos os dados serem coletados por um único método e o mesmo respondente preencher todas as variáveis (independentes, moderadora, mediadora e dependente), tem-se o problema do viés comum do método (CMB) (Podsakoff \& Organ, 1986). Para minimizar o CMB, incluiu-se uma carta de apresentação com instruções detalhadas e fez-se uma disposição clara e concisa dos itens da escala (Podsakoff, Mackenzie, \& Podsakoff, 2012). Após a coleta de dados, efetuou-se o teste de fator único de Harman, que resultou em $30,46 \%$ de variância total explicada, portanto abaixo do limiar de 50\% (Podsakoff \& Organ, 1986). Adicionalmente, efetuou-se o teste de viés de não resposta (decorrente do viés temporal), por meio do procedimento de Armstrong e Overton (1977), com o teste de Levene para igualdade de variâncias e o teste t para igualdade das médias entre os 10 primeiros e 10 últimos respondentes, mas não foram encontradas diferenças significativas nas respostas (valores-p maiores que 0,05). Assim, o CMB e o viés de não resposta não figuram um problema.

\section{Procedimentos de análise}

Na análise dos dados, aplicou-se a técnica de SEM-PLS, no software SmartPLS 3. Por essa técnica, faz-se a análise de caminhos para as relações propostas (Hair, Hult, Ringle, \& Sarstedt, 2016). Além dos efeitos diretos, são observadas análises de mediação (efeito indireto de uma variável independente em uma dependente pela variável mediadora) e moderação (fortalecimento ou enfraquecimento de uma relação pela interação de uma variável moderadora) (Hair et al., 2016).

No modelo de mensuração, foram excluídos dois itens do construto do engajamento no trabalho e um item da insegurança no trabalho, para ajuste do modelo. Pressupostos de validade discriminante, validade convergente, confiabilidade e ausência de multicolinearidade entre os preditores são observados (Hair et al., 2016). A significância foi calculada por bootstrapping de 5 mil reamostragens e, na moderação, utilizou-se o critério de dois estágios (Hair et al., 2016).

No tamanho mínimo de amostra para assegurar acurácia e robustez da SEM-PLS, duas abordagens foram observadas. A primeira consiste em a amostra mínima ser superior a 10 vezes o número máximo de relações (paths) analisadas no modelo estrutural (Hair et al., 2016). Nessa perspectiva, o número mínimo amostral seria $10 * 6$, o que equivale a 60 casos. 0 segundo aspecto tange ao uso do software $\mathrm{G}$ *Power 3.1, no qual se considera: poder do teste (Poder $=1-$ probabilidade de erro do tipo I) mínimo de o,8o (Cohen, 1988), tamanho do efeito (f2) médio de 0,15 e o maior número de variáveis conectadas a uma dependente (cinco variáveis ligadas ao engajamento no trabalho, para esse caso) (Ringle, Silva, \& Bido, 2014), o que resultou no mínimo de 92 casos necessários. Dessa forma, a amostra de 181 respondentes atende ambos os parâmetros.

Como análise complementar, fez-se uso da fsQCA, instrumentalizada no software fsQCA 3.0 (Ragin, 2008). Estudos pregressos sugerem o uso da SEM-PLS e, na sequência, a aplicação da fsQCA (Rihoux \& Ragin, 2009; Woodside, 2013), dada a complementaridade dos resultados das duas técnicas (Crespo et al., 2019; Kaya et al., 2020). A fsQCA permite a 
identificação de condições causais que promovem o sucesso da variável dependente, por meio de soluções que contribuem para o mesmo resultado desejado (equifinalidade) (Fiss, 2011; Ragin, 2008). Ademais, torna-se possível identificar as configurações das variáveis necessárias ou suficientes para promover o sucesso da variável dependente, por meio de combinações causais que antecedem a variável de interesse (Rihoux \& Ragin, 2009; Woodside, 2013).

\section{Tabela 1. Modelo de mensuração}

\begin{tabular}{|c|c|c|c|c|c|c|c|c|c|}
\hline & Construtos & $a$ & $\rho_{A}$ & CR & AVE & 1 & 2 & 3 & 4 \\
\hline 1 & CVI & 0,893 & 0,919 & 0,925 & 0,754 & 0,868 & 0,210 & 0,134 & 0,280 \\
\hline 2 & IT & 0,873 & 0,896 & 0,921 & 0,795 & $-0,205$ & 0,891 & 0,045 & 0,273 \\
\hline 3 & $\mathrm{Cl}$ & 0,932 & 0,942 & 0,957 & 0,880 & 0,118 & 0,022 & 0,938 & 0,249 \\
\hline 4 & ET & 0,836 & 0,877 & 0,901 & 0,752 & 0,248 & $-0,236$ & 0,230 & 0,867 \\
\hline
\end{tabular}

Nota: Os coeficientes na diagonal inferior/esquerda representam o critério de Fornell-Larcker, enquanto os coeficientes na diagonal superior/direita representam o critério HTMT; valores em negrito correspondem à raiz quadrada da AVE.

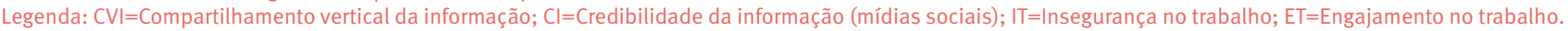

Os construtos denotam a pertinência de consistência interna, tanto pelo âmbito da carga fatorial dos itens $(\geq 0,708)$, alpha de Cronbach (a) $(\geq 0,70)$, Composite reliability (CR) $(\geq 0,70)$ como pelo critério de rho_A ( $\rho A)$, com índices acima de 0,70. Pelo viés da validade convergente, as Average Variance Extracted (AVE) são adequadas $(\geq 0,50)$. Pela ótica da validade discriminante, a

\section{ANÁLISE DOS DADOS}

\section{Resultados da PLS-SEM}

\section{Modelo de mensuração}

No modelo de mensuração (Tabela 1), são avaliadas a consistência interna (confiabilidade) e validades (convergente e discriminante) (Hair, Risher, Sarstedt, \& Ringle, 2019).

\section{Modelo estrutural}

Observada adequação satisfatória do modelo de mensuração, procedeu-se à análise do modelo estrutural (Tabela 2). Apresentam-se três painéis com análises de caminhos e um com poder preditivo das variáveis endógenas e ajustamento do modelo.

\section{Tabela 2. Análise de caminhos}

\begin{tabular}{|c|c|c|c|}
\hline Painéis & & \multicolumn{2}{|c|}{ Variáveis dependentes } \\
\hline \multicolumn{4}{|c|}{ Painel A - Efeitos diretos } \\
\hline Independentes & & Insegurança no trabalho & Engajamento no trabalho \\
\hline IT & & - & $-0,205^{\star \star \star}$ \\
\hline $\mathrm{Cl}$ & & - & $0,218^{* \star *}$ \\
\hline \multicolumn{4}{|c|}{ Painel B - Efeitos indiretos específicos (mediação) } \\
\hline CVI & IT & & $0,042^{*}$ \\
\hline \multicolumn{4}{|c|}{ Painel C - Efeitos de interação (moderação) } \\
\hline Independentes & Moderadora & & Engajamento no trabalho \\
\hline CVI & $\mathrm{Cl}$ & & 0,092 \\
\hline IT & $\mathrm{Cl}$ & & $0,123^{*}$ \\
\hline \multicolumn{4}{|c|}{ Painel D - Capacidade preditiva e ajustamento do modelo } \\
\hline \multicolumn{2}{|l|}{ SRMR } & \multicolumn{2}{|c|}{0,069} \\
\hline
\end{tabular}

Nota: ${ }^{*} p<0,10 ;{ }^{* *} p<0,05 ;{ }^{* * *} p<0,01$

Legenda: $\mathrm{CVI}=$ Compartilhamento vertical da informação; $\mathrm{Cl}=$ Credibilidade da informação (mídias sociais); IT=Insegurança no trabalho; ET=Engajamento no trabalho. 
Atesta-se pela ausência de multicolinearidade, pois a maior Variance Inflation Fator (VIF) dos construtos independentes foi 1,116, portanto abaixo do limiar de 3 (Hair et al., 2019). Acerca da redundância geral do modelo $\left(\mathrm{Q}^{2}\right)$ dos construtos dependentes, os índices acima de zero são aceitáveis (Hair et al., 2019). Quanto ao coeficiente de determinação $\left(R^{2}\right)$, a variância explicada da insegurança no trabalho é de pequena $(0,02)$ a média $(0,13)$, e, para o engajamento no trabalho, é de média $(0,13)$ a grande $(0,26)(C o h e n, 1988)$. Ademais, por meio da Standardized Root Mean Square Residual (SRMR), evidencia-se o ajustamento do modelo, no qual o coeficiente está abaixo do limite de 0,08 (Henseler, Hubona, \& Ray, 2016). Dessa forma, o modelo estrutural apresenta pressupostos satisfatórios. Assim, os resultados fornecem suporte estatístico para não rejeitar as hipóteses $\mathrm{H}_{a}, \mathrm{H} 2_{\mathrm{a}}, \mathrm{H} 2_{b}, \mathrm{H} 2_{c}$ e $\mathrm{H} 3_{\mathrm{a}}$.

\section{Variáveis de controle}

Para averiguar possíveis diferenças entre grupos da amostra, tempo no cargo e idade dos funcionários foram controlados (Tabela 3), mediante criação de subgrupos.

\section{Tabela 3. Análise de subgrupos}

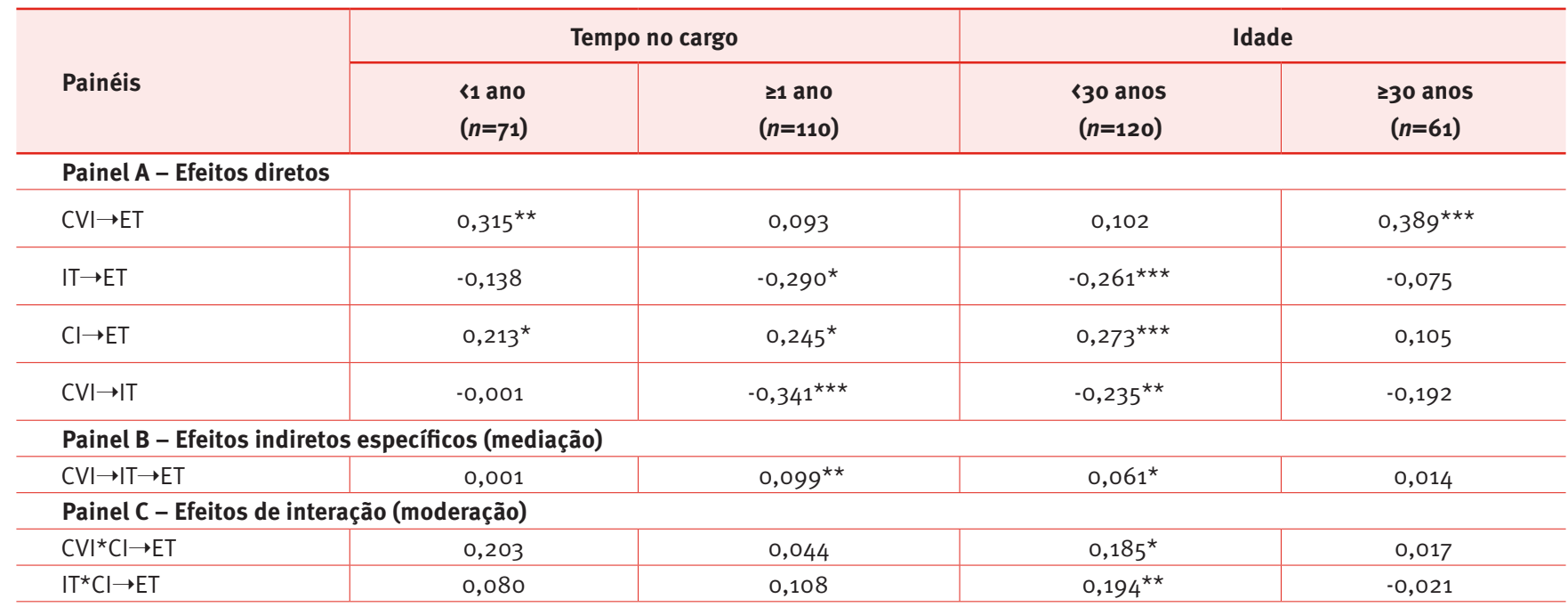

Nota: ${ }^{*} p<0,10 ;{ }^{* *} p<0,05 ;{ }^{* * *} p<0,01$.

Legenda: $\mathrm{CVI}=$ Compartilhamento vertical da informação; $\mathrm{Cl}=$ Credibilidade da informação (mídias sociais); IT=Insegurança no trabalho; $\mathrm{ET}=$ Engajamento no trabalho.

\section{Resultados fsQCA}

\section{Calibração}

A primeira etapa para análise fsQCA consiste na calibração das variáveis, de modo a transformar em conjuntos fuzzy, com valores de o a 1. Para a calibração, são definidas três âncoras: full membership (95\%), crossover point (50\%) e full non-membership (5\%) (Ragin, 2008). Para as variáveis mensuradas com escala tipo Likert de 7 pontos, essas âncoras foram fixadas respectivamente em 6, 4 e 2 (Ordanini, Parasuruman, \& Rubera, 2014). As variáveis tempo no cargo e idade foram calibradas de maneira dicotômica (crisp-set), conforme subgrupos definidos na metodologia.

\section{Condições necessárias e suficientes}

O segundo passo consistiu na análise das variáveis sempre necessárias (consistência $\geq 0,90$ ) e quase sempre necessárias (consistência $\geq 0,80$ ) (Ragin, 2008). Para promover alto engajamento no trabalho, o compartilhamento vertical da informação mostra-se quase sempre necessário $(0,824)$ e a ausência de insegurança no trabalho vislumbra-se sempre necessária $(0,922)$. Na terceira etapa, uma tabela verdade é originada, com todas as combinações possíveis. Para tanto, o limiar de consistência das soluções foi de o,80 (Ragin, 2008).

O software fsQCA gera três tipos de soluções: complexas, parcimoniosas e intermediárias. Neste estudo, optou-se pelo uso das soluções intermediárias, uma vez que é superior às outras, visto que não permite a remoção das condições necessárias (Ragin, 2017). Após a análise das condições necessárias, na Tabela 4 apresenta-se a análise de suficiência, ou seja, quais combinações de condições são suficientes para promover o alto engajamento no trabalho. Denota-se adequação da consistência geral da solução $(\geq 0,80)$ e ampla cobertura geral, o que sugere explicação de grande proporção do resultado. 
Tabela 4. Solução intermediária

\begin{tabular}{l|c|c}
\hline \multirow{2}{*}{ Construtos } & \multicolumn{2}{|c}{ Soluções para alto engajamento no trabalho } \\
\cline { 2 - 3 } & $S_{1}$ & $S_{2}$ \\
\hline Compartilhamento vertical da informação & $\otimes$ & $\otimes$ \\
\hline Insegurança no trabalho & $\otimes$ & 0 \\
\hline Credibilidade da informação (Mídias sociais) & & 0,773 \\
\hline Tempo no cargo & & 0,167 \\
\hline Idade & 0,493 & 0,439 \\
\hline Cobertura bruta & 0,048 & 0,926 \\
\hline Cobertura única & 0,909 & 0,935 \\
\hline Consistência & & 0,954 \\
\hline Cobertura geral da solução & & 0,896 \\
\hline Consistência geral da solução & & 0 \\
\hline
\end{tabular}

Nota: (i) Círculo preto ( ) representa a presença da condição; (ii) círculo branco com x ( $\otimes$ ) representa a ausência da condição; (iii) a não existência de círculos significa que tal condição é indiferente para a solução; esta tabela exclui as soluções com cobertura única muito baixa (menor que o,oo), similarmente a Miquel-Romero, Frasquet e Molla-Descals (2020).

Apesar de o compartilhamento vertical da informação e a ausência da insegurança no trabalho serem condições quase sempre e sempre necessárias, respectivamente, elas não são condições suficientes de maneiras independentes. Nesse contexto, três soluções $(S)$ são suficientes para promover o alto engajamento no trabalho: $S_{1}=$ ausência de insegurança no trabalho e credibilidade da informação; $S_{2}=$ presença de compartilhamento vertical da informação e ausência de insegurança no trabalho; e $S_{3}=$ presença de credibilidade da informação e ausência de idade (integrar o grupo com menos de 30 anos). As demais variáveis para cada solução são indiferentes.

\section{Discussão dos resultados}

Os resultados da pesquisa revelaram efeito negativo direto da insegurança no trabalho no engajamento no trabalho, fato que permite suportar $\mathrm{a} \mathrm{H}_{1}$. Para promover alto engajamento no trabalho, a ausência de insegurança no trabalho é uma condição necessária, além de ser suficiente em duas das três soluções obtidas (S1 e S2). Esse achado corrobora estudos prévios (Ali, Ali, Grigore et al., 2020; Guarnaccia et al., 2018; Karatepe et al., 2020). Em parte, a pandemia de Covid-19 propulsiona essa situação (Baker, 2020; Blake et al., 2010; Hamouche, 2020).

$\mathrm{A} \mathrm{H} 2$ a pode ser aceita, uma vez que existe efeito direto do compartilhamento vertical da informação no engajamento no trabalho. Ademais, o compartilhamento vertical da informação denota ser quase sempre necessário e suficiente em uma (S2) e indiferente em duas combinações causais ( $S_{1}$ e $S_{3}$ ) para alto engajamento no trabalho. Infere-se que, no período da pandemia de Covid-19, o compartilhamento vertical da informação se apresenta como um importante mecanismo gerencial na promoção de interação entre gestores e funcionários (Macinati et al., 2016; Parker \& Kyj, 2006), o que reflete no engajamento no trabalho (Baiman \& Evans, 1983; Karasek, 1979; Locke et al., 1986; Penno, 1984).

Para a ${ }_{2}$, há evidências de efeito negativo direto do compartilhamento vertical da informação na insegurança no trabalho, o que suporta a hipótese. Destarte, a comunicação entre gestores e funcionários, no âmbito da tomada de decisão ou do manifesto compartilhamento vertical de informações financeiras e não financeiras, mostra-se como um pertinente mecanismo que atua na mitigação da insegurança no trabalho dos funcionários (Vander Elst et al., 2010; Huang et al., 2012). Infere-se ainda o papel fundamental do compartilhamento vertical da informação no contexto da pandemia de Covid-19 (Hamouche, 2020), de modo a reduzir a assimetria informacional e a insegurança no trabalho (Darvishmotevali \& Ali, 2020; Lau \& Roopnarain, 2014; Nouri \& Parker, 1998).

$\mathrm{A} \mathrm{H}_{2}$ c é suportada, pois existe efeito indireto positivo do compartilhamento vertical da informação no engajamento no trabalho por meio da insegurança no trabalho. Desse modo, existe mediação parcial, uma vez que a relação direta também é estatisticamente significante (Bido \& Silva, 2019). Pode-se inferir 
que o compartilhamento vertical da informação, além de mitigar a insegurança no trabalho, por meio desta exerce efeito indireto positivo no engajamento no trabalho. Esse achado é corroborado em S2, no qual a presença do compartilhamento vertical da informação e a ausência da insegurança no trabalho fazem parte da solução suficiente para alto engajamento no trabalho, que contempla a maior proporção de casos (funcionários), tanto como combinação exclusiva (cobertura única) como compartilhada com as demais condições (cobertura bruta). Dessa maneira, vislumbra-se o pertinente papel do compartilhamento vertical da informação em detrimento da insegurança (Darvishmotevali \& Ali, 2020; Lau \& Roopnarain, 2014; Nouri \& Parker, 1998; Penno, 1984) e provimento do engajamento (Baiman \& Evans, 1983; Karasek, 1979; Locke et al., 1986).

$\mathrm{A} \mathrm{H}_{3}$, pode ser aceita, visto que existe efeito moderador positivo da credibilidade da informação (mídias sociais) na relação da insegurança no trabalho com o engajamento no trabalho. A interação é ilustrada na Figura 2.

\section{Figura 2. Efeito moderador}

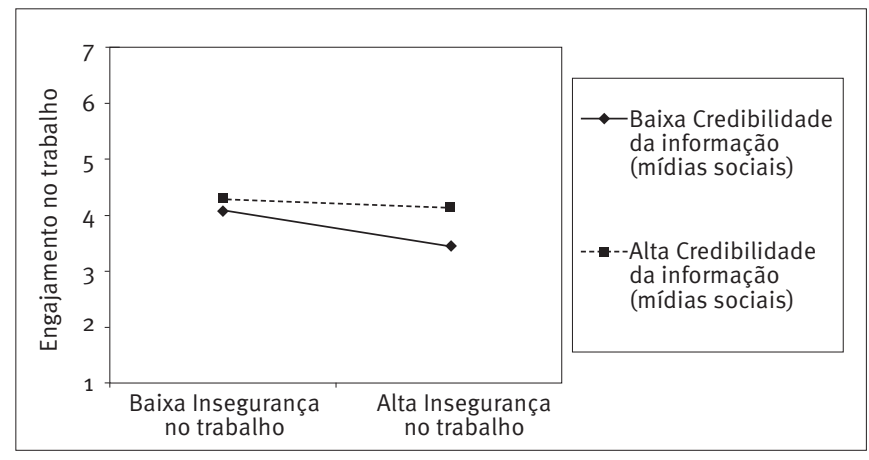

Observa-se que, sob cenários de baixa insegurança no trabalho, existe pouca variação da percepção de credibilidade da informação (mídias sociais) em relação ao engajamento no trabalho. No entanto, em cenários de alta insegurança no trabalho, à medida que diminui a credibilidade da informação, o engajamento no trabalho decresce. Assim, a credibilidade da informação pode agir em detrimento dessa situação (Men \& Yue, 2019; Mishra et al., 2014), pois, conforme aumenta, promove a minimização do impacto da insegurança no engajamento no trabalho, principalmente em cenários de alta insegurança no trabalho.

Argumenta-se que, no contexto de baixa credibilidade da informação sobre a pandemia de Covid-19, os funcionários podem apresentar maior medo, estresse e problemas de saúde mental (Brooks et al., 2018; Gao et al., 2020; Hamouche, 2020; Shigemura et al., 2020). A baixa percepção de credibilidade da informação aparentemente agrava a relação da insegurança no trabalho com o engajamento no trabalho (Saks, 2006). Por outro lado, em cenários de alta credibilidade da informação sobre a pandemia de Covid-19, os funcionários podem apresentar menores níveis de apreensão (Hamouche, 2020) e, portanto, pouco refletem na relação da insegurança no trabalho com o engajamento no trabalho.

A $\mathrm{H}_{3}$, que prevê efeito moderador positivo da credibilidade da informação (mídias sociais) na relação do compartilhamento vertical da informação com o engajamento no trabalho, não foi suportada estatisticamente. Esse achado indica que a credibilidade da informação sobre a pandemia de Covid-19, ancorada nas mídias sociais, não exerce interferência na forma como o compartilhamento vertical da informação se relaciona com o engajamento no trabalho. Depreende-se que o uso dos mecanismos gerenciais pela comunicação interna não está sofrendo impactos da comunicação externa (Verčič et al., 2012; Welch et al., 2012), mas sim que esse impacto está refletindo exclusivamente na relação da insegurança com o engajamento no trabalho $\left(\mathrm{H}_{3} \mathrm{a}\right)$, ou seja, a partir do momento em que isso não pode ser totalmente controlável pela gestão da startup.

O grupo mais jovem ( 30 anos) de respondentes revelou-se mais sensível em relação à credibilidade da informação (mídias sociais) sobre a pandemia de Covid-19, uma vez que essa modera tanto a relação do compartilhamento vertical da informação como a da insegurança no trabalho em relação ao engajamento no trabalho. Complementarmente, a $S_{3}$ evidencia que a ausência de idade (no caso, esse grupo mais jovem), juntamente com a percepção de credibilidade da informação, se configura como uma condição para alto engajamento no trabalho. Esse achado tem amparo na literatura, uma vez que maior percepção de credibilidade da informação implica menor problema psicológico e maior engajamento no trabalho (Blake et al., 2010; Brooks et al., 2018; Gao et al., 2020; Hamouche, 2020; Shigemura et al., 2020).

Para os grupos com mais tempo no cargo ( $\geq 1$ ano) e mais jovens ('30 anos), o compartilhamento vertical da informação não afeta diretamente o engajamento no trabalho, mas, por meio da mitigação da insegurança no trabalho, exerce efeito indireto, ou seja, ocorrem mediações totais (Bido \& Silva, 2019). Por sua vez, para os grupos com menor tempo no cargo ( $₫ 1$ ano) e mais velhos ( $\geq 30$ anos), não existe mediação, mas o compartilhamento vertical da informação influencia diretamente o engajamento no trabalho. Esses achados denotam que, dependendo do tempo no cargo ou da idade, os funcionários percebem essas variáveis de diferentes formas, porém, para todos os subgrupos, o compartilhamento vertical da informação possui efeito direto ou indireto no 
engajamento no trabalho, o que ressalta sua pertinência como mecanismo gerencial (Karasek, 1979; Macinati et al., 2016; Parker \& Kyj, 2006; Penno, 1984).

\section{CONSIDERAÇÕES FINAIS}

\section{Conclusões}

O estudo apresenta subsídios para concluir que, independentemente da idade ou tempo no cargo, o compartilhamento vertical da informação promove o engajamento no trabalho, seja de maneira direta ou indireta (por meio da mitigação da insegurança no trabalho). Nisso, o compartilhamento vertical da informação e ausência da insegurança no trabalho são quase sempre e sempre necessárias (respectivamente), bem como são solução suficiente para promover alto engajamento no trabalho, que contempla a maioria dos casos (funcionários). Singularmente, em cenários de alta insegurança no trabalho, a credibilidade da informação (mídias sociais) sobre a pandemia de Covid-19 assume importante papel na redução do impacto negativo no engajamento no trabalho dos funcionários da startup de serviços financeiros investigada.

\section{Implicações teóricas}

Embora estudos pregressos tenham examinado antecedentes da insegurança e engajamento no trabalho (Darvishmotevali \& Ali, 2020; Guarnaccia et al., 2018; Karatepe et al., 2020; Richter \& Näswall, 2019), aspectos como mecanismos gerenciais que visem à mitigação da insegurança e maximização do engajamento no trabalho ainda são necessários para a compreensão de tal fenômeno. A partir dessa lacuna, o presente estudo demonstra como o compartilhamento vertical da informação (financeira e não financeira) pode tornar-se um mecanismo de gestão para as organizações, no caso específico desta single entity para a maior startup brasileira de serviços financeiros, principalmente em cenário de incerteza como o observado no período da pandemia de Covid-19.

De acordo com o estudo de revisão da literatura realizado por Hamouche (2020), a maioria das pesquisas sobre o coronavírus concentra-se em aspectos da saúde física dos indivíduos, em que problemas da saúde mental parecem ter menor enfoque. Ainda conforme Hamouche (2020), os problemas mentais e a insegurança no trabalho podem estar interligados. Nesse contexto, conforme evidenciado por Gao et al. (2020), a falta de credibilidade da informação nas mídias sociais agrava a saúde mental da população, incluídos os trabalhadores. Dessa forma, as implicações oriundas deste estudo contribuem ao evidenciar que a (não) credibilidade da informação sobre a pandemia de Covid-19, nas mídias sociais, tende a agravar a relação da insegurança com o engajamento no trabalho.

\section{Implicações gerenciais}

Apesar de diversas empresas, em especial empresas inovadoras como as startups, estarem ameaçadas de sobrevivência e continuidade dos negócios neste período de pandemia da Covid-19 (Kuckertz et al., 2020), o presente estudo evidenciou que o compartilhamento vertical da informação pode ser um importante mecanismo na mitigação da insegurança e aumento do engajamento no trabalho dos funcionários. Ademais, a gestão deve levar em conta o impacto da credibilidade da informação nas mídias sociais, pela ótica dos funcionários. Para tanto, políticas de comunicação interna podem auxiliar nesse intento (Mishra et al., 2014; Verčič et al., 2012; Welch, 2012). Os subsídios deste estudo apresentam implicações para os gestores e funcionários de startups, o que pode auxiliar a continuidade dos negócios. Uma vez garantida a sua manutenção no mercado, as startups podem explorar novas oportunidades decorrentes de períodos de crise (Brem, Nylund, \& Viardot, 2020).

\section{Limitações e sugestões}

O estudo apresenta alguns fatores limitantes, que podem representar oportunidades para novas pesquisas. Primeiro, a generalização dos achados deve ser parcimoniosa em função das próprias delimitações da pesquisa. Segundo, na instrumentalização do construto de credibilidade da informação, houve a generalização de mídias sociais, sem controle específico, visto que diferentes mídias sociais (exs.: Facebook, Twitter, WhatsApp e YouTube) podem apresentar diferentes níveis de credibilidade e disseminação de fake news (Brennen, Simon, Howard, \& Nielsen, 2020). Terceiro, entre os mecanismos gerenciais, o estudo contemplou exclusivamente o compartilhamento vertical da informação; outros instrumentos de interação entre gestores e funcionários podem ser investigados, como os sistemas de controle gerencial. Quarto, o estudo utilizou apenas uma técnica para verificar a possível presença do CMB, podendo estudos futuros considerar outras ponderações, como o uso de variável marcadora. 


\section{REFERÊNCIAS}

Ali, M., Ali, I., Albort-Morant, G., \& Leal-Rodríguez, A.L. (2020). How do job insecurity and perceived well-being affect expatriate employees' willingness to share or hide knowledge? International Entrepreneurship and Management Journal, 1-26. doi:10.1007/ s11365-020-00638-1

Ali, I., Ali, M., Grigore, G., Molesworth, M., \& Jin, Z. (2020). The moderating role of corporate reputation and employee-company identification on the work-related outcomes of job insecurity resulting from workforce localization policies. Journal of Business Research, 117, 825-838. doi:10.1016/j.jbusres.2019.02.060

Armstrong, J. S., \& Overton, T. S. (1977). Estimating nonresponse bias in mail surveys. Journal of Marketing Research, 14(3), 396-402. doi:10.1177/002224377701400320

Baiman, S., \& Evans, J. H. (1983). Pre-decision information and participative management control systems. Journal of Accounting Research, 21(2), 371-395. doi:10.2307/2490780

Baker, M. G. (2020). Nonrelocatable occupations at increased risk during pandemics: United States, 2018. American Journal of Public Health, 110 (8), 1126-1132. doi:10.2105/ajph.2020.305738

Bao, Y., Sun, Y., Meng, S., Shi, J., \& Lu, L. (2020). 2019-nCoV epidemic: Address mental health care to empower society. The Lancet, 395(10224), 37-38. doi:10.1016/S0140-6736(20)30309-3

Battaglia, M. (2008). Purposive sample. In P. J. Lavrakas (Ed.), Encyclopedia of survey research methods (pp. 645-647). Thousand Oaks, USA: SAGE Publications. doi:10.4135/9781412963947.n419.

Bido, D. S., \& Silva, D. (2019). SmartPLS 3: Especificação, estimação, avaliação e relato. Administração: Ensino e Pesquisa, 20(2), 1-31. doi:10.13058/raep.2019.v2on2.154

Blake, K. D., Blendon, R. J., \& Viswanath, K. (2010). Employment and compliance with pandemic influenza mitigation recommendations. Emerging Infectious Diseases, 16(2), 212-218. doi:10.3201/ eid1602.090638

Bontcheva, K., Gorrell, G., \& Wessels, B. (2013). Social media and information overload: Survey results. arXiv. Retrieved from https:// arxiv.org/abs/1306.0813

Brem, A., Nylund, P., \& Viardot, E. (2020). The impact of the 2008 financial crisis on innovation: A dominant design perspective. Journal of Business Research, 110, 360-369. doi:10.1016/j. jbusres.2020.01.048

Brennen, J. S., Simon, F. M., Howard, P. N., \& Nielsen, R. K. (2020). Types, sources, and claims of Covid-19 misinformation. Reuters Institute. Retrieved from https://reutersinstitute.politics.ox.ac.uk/typessources-and-claims-covid-19-misinformation

Brooks, S. K., Dunn, R., Amlôt, R., Rubin, G. J., \& Greenberg, N. (2018). A systematic, thematic review of social and occupational factors associated with psychological outcomes in healthcare employees during an infectious disease outbreak. Journal of Occupational and Environmental Medicine, 60(3), 248-257. doi:10.1097/ JOM.0000000000001235

Carraro, W. B. W. H., Meneses, R., \& Brito, C. (2019). Combinação de categorias de práticas de controle de gestão para o alto desempenho de start-ups. Revista Brasileira de Gestão de Negócios, 21(4), 861878. doi:10.7819/rbgn.v21i5.4022
Cohen, J. (1988). Statistical power analysis for the behavioral sciences (2. ed.). New York: Psychology Press.

Crespo, N. F., Rodrigues, R., Samagaio, A., \& Silva, G. M. (2019). The adoption of management control systems by start-ups: Internal factors and context as determinants. Journal of Business Research, 101, 875-884. doi:10.1016/j.jbusres.2018.11.020

Darvishmotevali, M., \& Ali, F. (2020). Job insecurity, subjective wellbeing and job performance: The moderating role of psychological capital. International Journal of Hospitality Management, 87, 102472. doi:10.1016/j.ijhm.2020.102472

Fiss, P. C. (2011). Building better causal theories: A fuzzy set approach to typologies in organization research. Academy of Management Journal, 54(2), 393-420. doi:10.5465/amj.2011.60263120

Gao, J., Zheng, P., Jia, Y., Chen, H., Mao, Y., Chen, S., ... Dai, J. (2020). Mental health problems and social media exposure during Covid-19 outbreak. PLoS One, 15(4), e0231924. doi:10.1371/journal. pone. 0231924

Goodell, J. W. (2020). Covid-19 and finance: Agendas for future research. Finance Research Letters, 35, 1-5. doi:10.1016/j.frl.2020.101512

Greenhalgh, L., \& Rosenblatt, Z. (1984). Job insecurity: Toward conceptual clarity. Academy of Management Review, 9(3), 438-448. doi:10.5465/amr.1984.4279673

Guarnaccia, C., Scrima, F., Civilleri, A., \& Salerno, L. (2018). The role of occupational self-efficacy in mediating the effect of job insecurity on work engagement, satisfaction and general health. Current Psychology, 37(3), 488-497. doi:10.1007/s12144-016-9525-0

Haddad, C., \& Hornuf, L. (2019). The emergence of the global fintech market: Economic and technological determinants. Small Business Economics, 53(1), 81-105. doi:10.1007/s11187-018-9991-x

Hair, J. F., Jr., Hult, G. T. M., Ringle, C., \& Sarstedt, M. (2016). A primer on partial least squares structural equation modeling (PLS-SEM) (2nd ed.). Los Angeles, USA: Sage.

Hair, J. F., Jr., Risher, J. J., Sarstedt, M., \& Ringle, C. M. (2019). When to use and how to report the results of PLS-SEM. European Business Review, 31(1), 2-24. doi:10.1108/EBR-11-2018-0203

Hamouche, S. (2020). Covid-19 and employees' mental health: Stressors, moderators and agenda for organizational actions. Emerald Open Research, 2(15), 1-13. doi:10.35241/emeraldopenres.13550.1

Henseler, J., Hubona, G., \& Ray, P. A. (2016). Using PLS path modeling in new technology research: Updated guidelines. Industrial Management \& Data Systems, 116(1), 1-19. doi:10.1108/IMDS-092015-0382

Huang, G. H., Niu, X., Lee, C., \& Ashford, S. J. (2012). Differentiating cognitive and affective job insecurity: Antecedents and outcomes. Journal of Organizational Behavior, 33(6), 752-769. doi:10.1002/ job.1815

Karasek, R. A., Jr. (1979). Job demands, job decision latitude, and mental strain: Implications for job redesign. Administrative Science Quarterly, 24(2), 285-308. doi:10.2307/2392498

Karatepe, O. M., Rezapouraghdam, H., \& Hassannia, R. (2020). Job insecurity, work engagement and their effects on hotel employees' non-green and nonattendance behaviors. International Journal of Hospitality Management, 87, 102472. doi:10.1016/j. ijhm.2020.102472 
Kaya, B., Abubakar, A. M., Behravesh, E., Yildiz, H., \& Mert, I. S. (2020). Antecedents of innovative performance: Findings from PLS-SEM and fuzzy sets (fsQCA). Journal of Business Research, 114, 278-289. doi:10.1016/j.jbusres.2020.04.016

Kim, L., Fast, S. M., \& Markuzon, N. (2019). Incorporating media data into a model of infectious disease transmission. PloS One, 14(2), e0197646. doi:10.1371/journal.pone.0197646

Kuckertz, A., Brändle, L., Gaudig, A., Hinderer, S., Reyes, C. A. M., Prochotta, A., ... Berger, E. S. (2020). Startups in times of crisis: A rapid response to the Covid-19 pandemic. Journal of Business Venturing Insights, 13(e00169), 1-13. doi:10.1016/j.jbvi.2020.e00169

Lau, C. M., \& Roopnarain, K. (2014). The effects of nonfinancial and financial measures on employee motivation to participate in target setting. The British Accounting Review, 46(3), 228-247. doi:10.1016/j. bar.2014.02.006

Locke, E. A., Schweiger, D. M., \& Latham, G. P. (1986). Participation in decision making: When should it be used? Organizational Dynamics, 14(3), 65-79. doi:10.1016/0090-2616(86)90032-X

Lübke, C. (2019). How self-perceived job insecurity affects health: Evidence from an age-differentiated mediation analysis. Economic and Industrial Democracy, 1-18. doi:10.1177/0143831X19846333

Macinati, M. S., Bozzi, S., \& Rizzo, M. G. (2016). Budgetary participation and performance: The mediating effects of medical managers' job engagement and self-efficacy. Health Policy, 120(9), 1017-1028. doi:10.1016/j.healthpol.2016.08.005

Men, L. R., \& Yue, C. A. (2019). Creating a positive emotional culture: Effect of internal communication and impact on employee supportive behaviors. Public Relations Review, 45(3), 101764. doi:10.1016/j. pubrev.2019.03.001

Miquel-Romero, M. J., Frasquet, M., \& Molla-Descals, A. (2020). The role of the store in managing postpurchase complaints for omnichannel shoppers. Journal of Business Research, 109, 288-296. doi:10.1016/j. jbusres.2019.09.057

Mishra, K., Boynton, L., \& Mishra, A. (2014). Driving employeeengagement: The expanded role of internal communications. Journal of Business Communication, 51(2), 183-202. doi:10.1177/2329488414525399

Muthukannan, P., Tan, B., Gozman, D., \& Johnson, L. (2020). The emergence of a fintech ecosystem: A case study of the Vizag Fintech Valley in India. Information \& Management, 57(8), 1-14. doi:10.1016/j. im.2020.103385

Nouri, H., \& Parker, R. J. (1998). The relationship between budget participation and job performance: The roles of budget adequacy and organizational commitment. Accounting, Organizations and Society, 23(5-6), 467-483. doi:10.1016/S0361-3682(97)00036-6

Ordanini, A., Parasuraman, A., \& Rubera, G. (2014). When the recipe is more important than the ingredients: A qualitative comparative analysis (QCA) of service innovation configurations. Journal of Service Research, 17(2), 134-149. doi:10.1177/1094670513513337

Parker, R. J., \& Kyj, L. (2006). Vertical information sharing in the budgeting process. Accounting, Organizations and Society, 31(1), 2745. doi:10.1016/j.aos.2004.07.005

Penno, M. (1984). Asymmetry of pre-decision information and managerial accounting. Journal of Accounting Research, 22(1), 177191. doi:10.2307/2490707
Podsakoff, P. M, MacKenzie, S. B, \& Podsakoff, N. P. (2012). Sources of method bias in social science research and recommendations on how to control it. Annual Review of Psychology, 63, 539-569. doi:10.1146/annurev-psych-120710-100452

Podsakoff, P. M, \& Organ, D. W. (1986). Self-reports in organizational research: Problems and prospects. Journal of Management, 12, 531544. doi:10.1177/014920638601200408

Ragin, C. C. (2008). Redesigning social inquiry: Fuzzy sets and beyond. Chicago, USA: University of Chicago Press.

Ragin, C. C. (2017). User's guide to fuzzy-set/qualitative comparative analysis. Irvine, USA: University of California, Department of Sociology.

Rai, A., Ghosh, P., Chauhan, R., \& Mehta, N. K. (2017). Influence of job characteristics on engagement: Does support at work act as moderator? International Journal of Sociology and Social Policy, 37(1/2), 86-105. doi:10.1108/IJSSP-10-2015-0106

Richter, A., \& Näswall, K. (2019). Job insecurity and trust: Uncovering a mechanism linking job insecurity to well-being. Work \& Stress, 33(1), 22-40. doi:10.1080/02678373.2018.1461709

Rihoux, B., \& Ragin, C.C. (2009). Configurational comparative methods: Qualitative comparative analysis (QCA) and related techniques (Vol. 51). Califórnia: SAGE Publications Inc.

Ringle, C. M., Silva, D., \& Bido, D. S. (2014). Modelagem de equações estruturais com utilização do SmartPLS. Revista Brasileira de Marketing, 13(2), 56-73. doi:10.5585/remark.v13i2.2717

Saks, A. M. (2006). Antecedents and consequences of employee engagement. Journal of Managerial Psychology, 21(7), 600-619. doi:10.1108/02683940610690169

Shigemura, J., Ursano, R. J., Morganstein, J. C., Kurosawa, M., \& Benedek, D. M. (2020). Public responses to the novel 2019 coronavirus (2019 $\mathrm{nCoV}$ ) in Japan: Mental health consequences and target populations. Psychiatry and Clinical Neurosciences, 74(4), 281. doi:10.1111/ pcn.12988

Stander, M. W., \& Rothmann, S. (2010). Psychological empowerment, job insecurity and employee engagement. SA Journal of Industrial Psychology, 36(1), 1-8. doi:10.4102/sajip.v36i1.849

Thompson, N., Wang, X., \& Daya, P. (2019). Determinants of news sharing behavior on social media. Journal of Computer Information Systems, 6o(6), 1-9. doi:10.1080/08874417.2019.1566803

Vander Elst, T., Baillien, E., Cuyper, N., \& Witte, H. (2010). The role of organizational communication and participation in reducing job insecurity and its negative association with work-related well-being. Economic and Industrial Democracy, 31(2), 249-264. doi:10.1177/0143831X09358372

Verčič, A. T., Verčič, D., \& Sriramesh, K. (2012). Internal communication: Definition, parameters, and the future. Public Relations Review, 38(2), 223-230. doi:10.1016/j.pubrev.2011.12.019

Walsh, G. S., \& Cunningham J. A. (2016). Business failure and entrepreneurship: Emergence, evolution and future research. Foundations and Trends $₫$ in Entrepreneurship, 12(3), 163-285. doi:10.1561/0300000063 
Welch, M. (2012). Appropriateness and acceptability: Employee perspectives of internal communication. Public Relations Review, 38(2), 246-254. doi:10.1016/j.pubrev.2011.12.017

Wiederhold, B. K. (2020). Social media use during social distancing: Cyberpsychology. Behavior and Social Networking, 23(5), 1-2. doi:10.1089/cyber.2020.29181.bkw

Witte, H. (2000). Arbeidsethos en jobonzekerheid: Meting en gevolgen voor welzijn, tevredenheid en inzet op het werk. In R. Bouwen, K. Witte, H. Witte, \& T. Taillieu (Eds.), Van groep naar gemeenschap (pp. 325-350). Leuven, Belgium: Garant.
Woodside, A. G. (2013). Moving beyond multiple regression analysis to algorithms: Calling for adoption of a paradigm shift from symmetric to asymmetric thinking in data analysis and crafting theory. Journal of Business Research, 66(4), 463-472. doi:10.1016/j. jbusres.2012.12.021

Yanagi, Y., Tahara, Y., Orihara, R., Sei, Y., \& Ohsuga, A. (2020). Fake news detection with generated comments for news articles (n.3190). EasyChair. Retrieved from https://easychair.org/publications/ preprint/sgzm

Zarocostas, J. (2020). How to fight an infodemic. The Lancet, 395(10225), 676. doi:10.1016/S0140-6736(20)30461-X

\section{CONTRIBUIÇÃO DOS AUTORES}

Os autores declaram que contribuíram igualmente no desenvolvimento do artigo, incluindo conceitualização e abordagem teórico-metodológica, revisão teórica (levantamento de literatura), coleta e análise dos dados, até a redação e revisão final. 